\title{
New results on eccentric connectivity indices of V-Phenylenic nanotube
}

\author{
Zaheer Ahmad ${ }^{\circ}$ |Maria Naseem ${ }^{b} \quad$ |Muhammad Kamran Jamilc ${ }^{\circ}$ |Muhammad Kamran

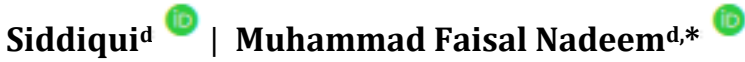

aKhwaja Fareed University of Engineering and Information Technology, Rahim Yar Khan, Pakistan

${ }^{b}$ Abdus Salam School of Mathematical Sciences, GC University, Lahore, Pakistan

'Department of Mathematics, Riphah Institute of Computing and Applied Sciences (RICAS), Riphah International University, Lahore, Pakistan

dDepartment of Mathematics, Comsats University Islamabad Lahore Campus

\author{
*Corresponding Author: \\ Muhammad Faisal Nadeem \\ Email: mfaisalnadeem@ymail.com \\ Tel.: +923454753701
}

\begin{abstract}
Topological index is a type of molecular descriptor calculated based on the molecular graph of a chemical compound. Topological indices are used for developing the quantitative structure activity relationships (QSARs) in which the biological activity or other properties of the molecules are correlated with their chemical structure. Eccentric connectivity indices are the well-known topological indices in this regards. In this research study, we computed some eccentric connectivity indices of the VPhenylenic nanotube VPHX[p;q], these are our results.
\end{abstract}

\section{KEYWORDS}

Molecular graph; eccentricity; ediz eccentric connectivity index; Augmented eccentric connectivity index; V-Phenylenic nanotube VPHX[p;q].

\section{Introduction}

Let $\mathrm{G}=(\mathrm{V} ; \mathrm{E})$ be a molecular graph, where $\mathrm{V}(\mathrm{G})$ is a non-empty set of vertices and $E(G)$ is a set of edges. Cardinality of the vertex set is said to be order of graph $\mathrm{G}$, denoted by $|\mathrm{V}(\mathrm{G})|$ and the cardinality of edge set is the size of graph, denoted by $|\mathrm{E}(\mathrm{G})|$. Number of edges incident with vertex $v$ is called the degree of vertex. The distance from $u$ to $v$, where $u ; v \in V(G)$, is defined as the length of the shortest path from $u$ to $v$, denoted by $d(u ; v)$. The eccentricity of a vertex $v \in V(G)$, denoted by $\epsilon(v)$, is the maximum distance between the $\mathrm{v}$ vertex to all other vertices i.e.

$$
\varepsilon(u)=\max \{d(u, v) \mid \forall u \in V(G)\}
$$

The topological index which is also referred to molecular descriptor is a real number which describes the properties of a certain chemical compounds. The study of topological indices on different chemical structures has been an attractive area of research for all graph theorist. Actually, it is a bridge between mathematics and chemistry. Simplest topological indices of a graph G are the order, size, degree of vertices $v$ and distance between vertices. Among the molecular descriptors, topological connectivity indices are very important and many of them have found applications as means to model chemical, pharmaceutical and other properties of the molecules. In 1975, oldest topological connectivity index of a connected graph G introduced by M. Randić, who showed this index to reflect the molecular branching [1-3]. Now, we call it Randić connectivity index R(G) is equal to $R(G)=\sum_{u v \in E(G)} \frac{1}{\sqrt{d(u) d(v)}}$ where $\mathrm{d}$ (v) denotes the degree of vertex $\mathrm{v}$ of $\mathrm{G}$.

The eccentric connectivity index of a graph G was proposed by Sharma, Goswami and Madan in [4], as $\zeta^{c}(G)=\sum_{v \in V(G)} d(v) \varepsilon(v)$ The eccentric connectivity index was successfully used for mathematical models of biological activities of diverse nature $[45,46,47,48]$. The prediction of the physico-chemical, 
pharmacological and toxicological properties of compounds directly from their molecular structure has become an important tool in pharmaceutical drug design. The parameters derived from a graph theoretic model of a chemical structure are being used not only in QSAR studies, but also in the environmental hazard assessment of chemicals. Eccentric connectivity index revealed a high degree of predictability of pharmaceutical properties, providing leads for developing safe and potent anti-HIV compounds [40-44, 49, 50]. Eccentric connectivity index is also proposed as a measure of branching in alkanes [51].

Gupta et al. [5] introduced the connective eccentric index for a graph

$$
C^{\zeta}(G)=\sum_{v \in V(G)} \frac{d(v)}{\varepsilon(v)}
$$

Recently, S. Ediz et al. defined Ediz eccentric connectivity index of $\mathrm{G}$, denoted by ${ }^{E} \zeta^{c}(G)$ and is defined as

$$
{ }^{E} \zeta^{c}(G)=\sum_{v \in V(G)} \frac{S_{v}}{\varepsilon(v)}
$$

where $S_{v}$ is the sum of degrees of all vertices adjacent to vertex $v[6,7]$. A generalization of eccentric connectivity index, known as augmented eccentric connectivity index of a graph G was proposed by Dureja and Madan in [8]: ${ }^{A} \zeta^{c}(G)=\sum_{v \in V(G)} \frac{M_{v}}{\varepsilon(v)}$ where $\mathrm{M}_{\mathrm{v}}$ is the product of degrees of all vertices adjacent to vertex v. For further details about these new connectivity indices see [9-29].

In this work, we extended the results of [30] and determined the exact formulas for Ediz and Augmented eccentric connectivity indices.

\section{Result and discussion}

In this study, we deal with the molecular graph of V-Phenylenic nanotube VPHX[p;q], where $p$ denotes the number of hexagons in a column and $\mathrm{q}$ denotes the number of hexagons in a row of the V-Phenylenic nanotube.

In this section, we computed the Ediz eccentric connectivity and augmented eccentric connectivity indices of V-Phenylenic nanotube VPHX[p;q]. For further details and more study about the V-Phenylenic nanotube VPHX[p;q] see [31-39]. Rao and Lakshmi calculated some eccentric connectivity index of V-Phenylenic nanotube [52]. In this research study we extend the results found in [52] and calculated above mention eccentric connectivity indices.

Figure 1 demonstrates the representation of V-Phenylenic nanotube VPHX[p;q]. As seen in Figure 1, the number of vertices and edges in $\mathrm{G}=\mathrm{VPHX}[\mathrm{p} ; \mathrm{q}]$ are equal to $6 \mathrm{pq}$ and $9 \mathrm{pq}-\mathrm{q}$, respectively. It is clear that there are $2 p$ vertices of degree 2, $6 p q-2 p$ vertices of degree 3 , We denote the set of vertices of degree two and degree three by $V_{2}$ and $V_{3}$, respectively. The graph of V-Phenylenic nanotube has $4 \mathrm{p}$ rows and q columns.

To compute the Ediz eccentric index and Augmented eccentric connectivity index of the V-Phenylenic nanotube VPHX[p;q], we need vertex partition of the $\mathrm{V}$-Phenylenic nanotube VPHX[p;q] based on degree sum and degree multiplication of neighbors vertices, respectively.

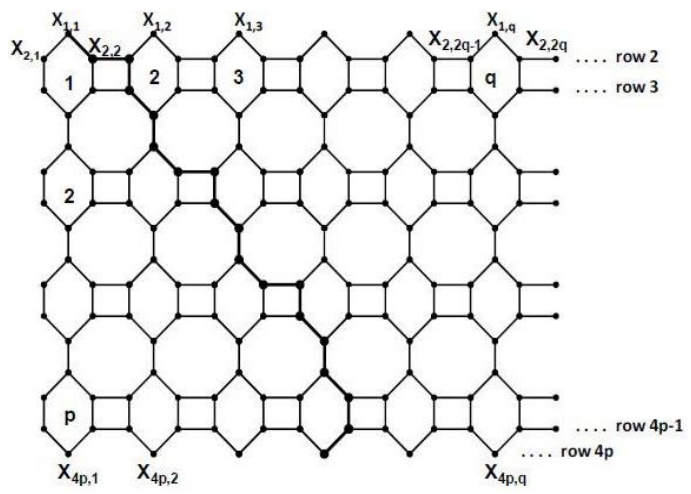

FIGURE 1 The molecular graph of VPhelylenic nanotube [30] 
TABLE 1 Vertex partition based on degree sum of neighbor vertices

\begin{tabular}{cc}
\hline $\begin{array}{c}S_{\mathbf{v}} \text { where } \mathbf{\epsilon} \boldsymbol{\epsilon} \text { VPHX } \\
{[\mathbf{p}, \mathbf{q}]}\end{array}$ & $\begin{array}{c}\text { Number of } \\
\text { Vertices }\end{array}$ \\
\hline 8 & $2 \mathrm{p}$ \\
9 & $4 \mathrm{p}$ \\
10 & $2 \mathrm{pq}$ \\
12 & $2 \mathrm{p}$ \\
13 & $2 \mathrm{pq}-2 \mathrm{p}$ \\
14 & $2 \mathrm{p}$ \\
15 & $2 \mathrm{pq}-2 \mathrm{p}$ \\
\hline
\end{tabular}

TABLE 2 Vertex partition based on product of degree of neighbor vertices

\begin{tabular}{cc}
\hline$M_{\mathbf{v}}$ where $\mathbf{\epsilon}$ & $\begin{array}{c}\text { Number of } \\
\text { Vertices }\end{array}$ \\
(VPHX [p,q]) & $2 \mathrm{p}$ \\
16 & $24 \mathrm{p}$ \\
20 & $2 \mathrm{p}$ \\
24 & $2 \mathrm{p}$ \\
72 & $2 \mathrm{pq}$ \\
25 & $2 \mathrm{pq}-2 \mathrm{p}$ \\
108 & $2 \mathrm{p}$ \\
100 & $2 \mathrm{pq}-2 \mathrm{p}$ \\
125 & \\
\hline
\end{tabular}

To compute the sum of degrees of all neighbors of vertices in the V-Phenylenic nanotube VPHX[p;q], we presented the vertex partitions based on degree sum with their cardinalities in Table 1.

To compute the multiplication of degrees of all neighboring vertices in the V-Phenylenic nanotube VPHX[p;q], we presented the vertex partitions based on product of degrees, with their cardinalities in Table 2.

Theorem 1. Let VPHX[p;q] be the graph of $\mathrm{V}$-Phenylenic nanotubes, then:

$$
\begin{aligned}
{ }^{E} \zeta^{c}(V P H X[p, q]) & = \begin{cases}100 & ; \text { when } p=1 \text { and } q=1 \\
66 q^{2}+44 q & ; \text { when } p=1 \text { and qis even } \\
66 q^{2}+22 q & ; \text { when } p=1 \text { and qisodd }\end{cases} \\
{ }^{A} \zeta^{c}(V P H X[p, q]) & = \begin{cases}198 & ; \text { when } p=1 \text { and } q=1 \\
135 q^{2}+90 q & ; \text { when } p=1 \text { and qis even } \\
135 q^{2}+45 q & ; \text { when } p=1 \text { and qis odd }\end{cases}
\end{aligned}
$$

Proof. In this case the graph has only 4 rows. The sum of degrees of neighboring vertices in $1^{\text {st }}$ and $4^{\text {th }}$ rows is 6 , whereas the sum of degrees of neighboring vertices in $2^{\text {nd }}$ and $3^{\text {rd }}$ rows is 8 . The product of degrees of neighboring vertices in $1^{\text {st }}$ and $4^{\text {th }}$ rows is 9 , whereas the product of degrees of neighboring vertices in $2^{\text {nd }}$ and $3^{\text {rd }}$ rows is 18.

Case 1. When $\mathrm{p}=1$ and $\mathrm{q}=1$ :

$$
\begin{aligned}
& { }^{E} \zeta^{c}(G)=\sum_{v \in V(G)} \frac{S_{v}}{\varepsilon(v)}=2[6(3)+2(8)(2)]=100, \\
& { }^{A} \zeta^{c}(G)=\sum_{v \in V(G)} \frac{M_{v}}{\varepsilon(v)}=2[9(3)+2(18)(2)]=198 .
\end{aligned}
$$

Case 2. When $\mathrm{p}=1$ and $\mathrm{q}$ is even:

In this case the eccentricity of every vertex in every row is $\left(\frac{3 q+2}{2}\right)$,

$$
\begin{aligned}
& { }^{E} \zeta^{c}(G)=\sum_{v \in V(G)} \frac{S_{v}}{\varepsilon(v)}= \\
& 2\left[6(q)\left(\frac{3 q+2}{2}\right)+8(2 q)\left(\frac{3 q+2}{2}\right)\right]=66 q^{2}+44 q, \\
& { }^{A} \zeta^{c}(G)=\sum_{v \in V(G)} \frac{M_{v}}{\varepsilon(v)}=2\left[9(q)\left(\frac{3 q+2}{2}\right)\right. \\
& \left.+18(2 q)\left(\frac{3 q+2}{2}\right)\right]=135 q^{2}+90 q .
\end{aligned}
$$

Case 3: When $\mathrm{p}=1$ and $\mathrm{q}$ is odd:

In this case the eccentricity of every vertex in every row is $\left(\frac{3 q+1}{2}\right)$, So,

$$
\begin{aligned}
& { }^{E} \zeta^{c}(G)=\sum_{v \in V(G)} \frac{S_{v}}{\varepsilon(v)}=2\left[6(q)\left(\frac{3 q+1}{2}\right)\right. \\
& \left.+8(2 q)\left(\frac{3 q+1}{2}\right)\right]=66 q^{2}+22 q, \\
& { }^{A} \zeta^{c}(G)=\sum_{v \in V(G)} \frac{M_{v}}{\varepsilon(v)}=2\left[9(q)\left(\frac{3 q+1}{2}\right)\right. \\
& \left.+18(2 q)\left(\frac{3 q+1}{2}\right)\right]=135 q^{2}+45 q .
\end{aligned}
$$

Theorem 2. Let VPHX[p;q] be the graph of VPhenylenic nanotubes, when $\mathrm{q} \leq \mathrm{p}, \mathrm{p}$ and $\mathrm{q}$ are both even, then we have

$$
\begin{aligned}
& { }^{E} \zeta^{c}(V P H X[p, q])=162 p^{2} q+27 p q^{2}-67 p q-5 q^{2}+14 q \\
& { }^{A} \zeta^{c}(V P H X[p, q])=486 p^{2} q-81 p q^{2}-369 p q-36 q^{2}+108 q
\end{aligned}
$$

Proof. In this case the graph has $4 p$ rows and the eccentricity of a vertex in $i^{\text {th }}$ row is same as the eccentricity of a vertex in $(4 p+1-i)^{\text {th }}$ row 
where $i=1,2, \ldots, 2 p$. . Let $\mathrm{x}_{\mathrm{i}}$ be a vertex in $\mathrm{i}^{\text {th }}$ row. The eccentricity of $\mathrm{X}_{\mathrm{i}}$ is $\varepsilon\left(x_{i}\right)=\left(\frac{8 p+q}{2}-i\right)$ where $i=1,2, \ldots, 2 p$. Here the sum of degrees of neighboring vertices in $1^{\text {st }}$ and $4^{\text {th }}$ rows is 6 , whereas the sum of degrees of neighboring vertices in $2^{\text {nd }}$ and $3^{\text {rd }}$ rows is 8 . The product of degrees of neighboring vertices in $1^{\text {st }}$ and $4^{\text {th }}$ rows is 9 , whereas the product of degrees of neighboring vertices in $2^{\text {nd }}$ and $3^{\text {rd }}$ rows is 18 . Hence:

$$
\begin{aligned}
& { }^{E} \zeta^{c}(G)=\sum_{v \in V(G)} \frac{S_{v}}{\varepsilon(v)}=2\left[6(q)\left(\frac{8 p+q}{2}-1\right)\right. \\
& +8(2 q)\left(\frac{8 p+q}{2}-2\right)+9(2 q)\left(\frac{8 p+q}{2}-3\right)+9(q)\left(\frac{8 p+q}{2}-4\right) \\
& +9(q)\left(\frac{8 p+q}{2}-5\right)+9(2 q)\left(\frac{8 p+q}{2}-6\right) \\
& +9(2 q)\left(\frac{8 p+q}{2}-7\right)+\cdots+9(q)\left(\frac{8 p+q}{2}-2 p\right) \\
& =162 p^{2} q+27 p q^{2}-67 p q-5 q^{2}+14 q \\
& { }^{A} \zeta^{c}(G)=\sum_{v \in V(G)} \frac{M_{v}}{\varepsilon(v)}=2\left[8(q)\left(\frac{8 p+q}{2}-1\right)+18(2 q)\left(\frac{8 p+q}{2}-2\right)\right. \\
& +27(2 q)\left(\frac{8 p+q}{2}-3\right)+27(q)\left(\frac{8 p+q}{2}-4\right) \\
& +27(q)\left(\frac{8 p+q}{2}-5\right)+27(2 q)\left(\frac{8 p+q}{2}-6\right)+27(2 q)\left(\frac{8 p+q}{2}-7\right) \\
& +\cdots+27(q)\left(\frac{8 p+q}{2}-2 p\right) \\
& =486 p^{2} q-81 p q^{2}-369 p q-36 q^{2}+108 q .
\end{aligned}
$$

Theorem 3. Let VPHX[p;q] be the graph of VPhenylenic nanotubes, when $\mathrm{q} \leq \mathrm{p}, \mathrm{p}$ is even and $\mathrm{q}$ is odd, then we have:

$$
\begin{aligned}
& { }^{E} \zeta^{c}(V P H X[p, q])=162 p^{2} q+27 p q^{2}-94 p q-5 q^{2}+19 q \\
& { }^{A} \zeta^{c}(V P H X[p, q])=486 p^{2} q-81 p q^{2}-434 p q-36 q^{2}+144 q
\end{aligned}
$$

Proof. In this case the graph has 4 rows and the eccentricity of a vertex in $i^{\text {th }}$ row is same as the eccentricity of a vertex in $(4 p+1-i)^{\text {th }}$ row where $i=1,2, \ldots, 2 p$. Let $\mathrm{x}_{\mathrm{i}}$ be a vertex in $\mathrm{i}^{\text {th }}$ row. The eccentricity of $x i$ is $\varepsilon\left(x_{i}\right)=\left(\frac{8 p+q-1}{2}-i\right) \quad$ where $i=1,2, \ldots, 2 p$.

Here the sum of degrees of neighboring vertices in $1^{\text {st }}$ and $4^{\text {th }}$ rows is 6 , whereas the sum of degrees of neighboring vertices in $2^{\text {nd }}$ and $3^{\text {rd }}$ rows is 8 . The product of degrees of neighboring vertices in $1^{\text {st }}$ and $4^{\text {th }}$ rows is 9 , whereas the product of degrees of neighboring vertices in $2^{\text {nd }}$ and $3^{\text {rd }}$ rows is 18 . Hence:

$$
\begin{aligned}
& { }^{E} \zeta^{c}(G)=\sum_{v \in V(G)} \frac{S_{v}}{\varepsilon(v)}=2\left[6(q)\left(\frac{8 p+q-1}{2}-1\right)\right. \\
& +8(2 q)\left(\frac{8 p+q-1}{2}-2\right)+9(2 q)\left(\frac{8 p+q-1}{2}-3\right) n \\
& +9(q)\left(\frac{8 p+q-1}{2}-4\right)+9(q)\left(\frac{8 p+q-1}{2}-5\right) \\
& +9(2 q)\left(\frac{8 p+q-1}{2}-6\right)+9(2 q)\left(\frac{8 p+q-1}{2}-7\right) \\
& \left.+\cdots+9(q)\left(\frac{8 p+q-1}{2}-2 p\right)\right] \\
& =162 p^{2} q+27 p q^{2}-94 p q-5 q^{2}+19 q \\
& { }^{A}(G)=\sum_{v \in V(G)} \frac{M_{v}}{\varepsilon(v)}=2\left[8(q)\left(\frac{8 p+q-1}{2}-1\right)\right. \\
& +18(2 q)\left(\frac{8 p+q-1}{2}-2\right)+27(2 q)\left(\frac{8 p+q-1}{2}-3\right) \\
& +27(q)\left(\frac{8 p+q-1}{2}-4\right)+27(q)\left(\frac{8 p+q-1}{2}-5\right) \\
& +27(2 q)\left(\frac{8 p+q-1}{2}-6\right)+27(2 q)\left(\frac{8 p+q-1}{2}-7\right) \\
& \left.+\cdots+27(q)\left(\frac{8 p+q-1}{2}-2 p\right)\right] \\
& +486 p^{2} q-81 p q^{2}-434 p q-36 q^{2}+144 q
\end{aligned}
$$

Theorem 4. Let VPHX[p;q] be the graph of VPhenylenic nanotubes, when $q \leq p, p$ is odd and $\mathrm{q}$ is even, then we have:

$$
\begin{aligned}
& { }^{E} \zeta^{c}(V P H X[p, q])=162 p^{2} q+27 p q^{2}-103 p q-14 q^{2}+5 q \\
& { }^{A} \zeta^{c}(V P H X[p, q])=486 p^{2} q+81 p q^{2}-369 p q-36 q^{2}+81 q
\end{aligned}
$$

Proof. In this case the graph has 4 rows and the eccentricity of a vertex in $i^{\text {th }}$ row is same as the eccentricity of a vertex in $(4 \mathrm{p}+1-\mathrm{i})^{\text {th }}$ row where $i=1,2, \ldots, 2 p$. Let $\mathrm{x}_{\mathrm{i}}$ be a vertex in $\mathrm{i}^{\text {th }}$ row. The eccentricity of $\mathrm{x}_{\mathrm{i}}$ is $\varepsilon\left(x_{i}\right)=\left(\frac{8 p+q}{2}-i\right)$ where $i=1,2, \ldots, 2 p$. Here the sum of degrees of neighboring vertices in $1^{\text {st }}$ and $4^{\text {th }}$ rows is 6 , whereas the sum of degrees of neighboring vertices in $2^{\text {nd }}$ and $3^{\text {rd }}$ rows is 8 . The product of degrees of neighboring vertices in $1^{\text {st }}$ and $4^{\text {th }}$ rows is 9 , whereas the product of degrees of neighboring vertices in $2^{\text {nd }}$ and $3^{\text {rd }}$ rows is 18. Hence: 


$$
\begin{aligned}
& { }^{E} \zeta^{c}(G)=\sum_{v \in V(G)} \frac{S_{v}}{\varepsilon(v)}=2\left[6(q)\left(\frac{8 p+q}{2}-1\right)\right. \\
& +8(2 q)\left(\frac{8 p+q}{2}-2\right)+9(2 q)\left(\frac{8 p+q}{2}-3\right) \\
& +9(q)\left(\frac{8 p+q}{2}-4\right)+9(q)\left(\frac{8 p+q}{2}-5\right) \\
& +9(2 q)\left(\frac{8 p+q}{2}-6\right)+9(2 q)\left(\frac{8 p+q}{2}-7\right) n \\
& +9(q)\left(\frac{8 p+q}{2}-8\right)+9(q)\left(\frac{8 p+q}{2}-9\right) \\
& \left.+\cdots+9(q)\left(\frac{8 p+q-1}{2}-2 p\right)\right] \\
& =162 p^{2} q+27 p q^{2}-103 p q-14 q^{2}+5 q \\
& +\zeta^{c}(G)=\sum_{v \in V(G)} \frac{M_{v}}{\varepsilon(v)}=2\left[8(q)\left(\frac{8 p+q}{2}-1\right)\right. \\
& +18(2 q)\left(\frac{8 p+q}{2}-2\right)+27(2 q)\left(\frac{8 p+q}{2}-3\right) \\
& +27(q)\left(\frac{8 p+q}{2}-4\right)+27(q)\left(\frac{8 p+q}{2}-5\right) \\
& +27(2 q)\left(\frac{8 p+q}{2}-6\right)+27(2 q)\left(\frac{8 p+q}{2}-7\right) \\
& +27(q)\left(\frac{8 p+q}{2}-8\right)+27(q)\left(\frac{8 p+q}{2}-9\right) \\
& \left.+\cdots+27(q)\left(\frac{8 p+q-1}{2}-2 p\right)\right] \\
& +p^{2} q+81 p q^{2}-369 p q-36 q^{2}+81 \\
& +96
\end{aligned}
$$

Theorem 5. Let VPHX[p;q] be the graph of VPhenylenic nanotubes, when $\mathrm{q} \leq \mathrm{p}, \mathrm{p}$ and $\mathrm{q}$ both are odd, then we have

$$
\begin{aligned}
& { }^{E} \zeta^{c}(V P H X[p, q])=162 p^{2} q+27 p q^{2}-94 p q-5 q^{2}+10 q \\
& { }^{A} \zeta^{c}(V P H X[p, q])=486 p^{2} q+81 p q^{2}-450 p q-36 q^{2}+117 q
\end{aligned}
$$

Proof. In this case the graph has 4 rows and the eccentricity of a vertex in $i^{\text {th }}$ row is same as the eccentricity of a vertex in $(4 \mathrm{p}+1-\mathrm{i})^{\text {th }}$ row where $i=1,2, \ldots, 2 p$. Let $\mathrm{x}_{\mathrm{i}}$ be a vertex in $\mathrm{i}^{\text {th }}$ row. The eccentricity of $x i$ is $\varepsilon\left(x_{i}\right)=\left(\frac{8 p+q-1}{2}-i\right)$ where $i=1,2, \ldots, 2 p$.

Here the sum of degrees of neighboring vertices in $1^{\text {st }}$ and $4^{\text {th }}$ rows is 6 , whereas the sum of degrees of neighboring vertices in $2^{\text {nd }}$ and $3^{\text {rd }}$ rows is 8 . The product of degrees of neighboring vertices in $1^{\text {st }}$ and $4^{\text {th }}$ rows is 9 , whereas the product of degrees of neighboring vertices in $2^{\text {nd }}$ and $3^{\text {rd }}$ rows is 18 . Hence

$$
\begin{aligned}
& { }^{E} \zeta^{c}(G)=\sum_{v \in V(G)} \frac{S_{v}}{\varepsilon(v)}=2\left[6(q)\left(\frac{8 p+q-1}{2}-1\right)\right. \\
& +8(2 q)\left(\frac{8 p+q-1}{2}-2\right)+9(2 q)\left(\frac{8 p+q-1}{2}-3\right) \\
& +9(q)\left(\frac{8 p+q-1}{2}-4\right)+9(q)\left(\frac{8 p+q-1}{2}-5\right) \\
& +9(2 q)\left(\frac{8 p+q-1}{2}-6\right)+9(2 q)\left(\frac{8 p+q-1}{2}-7\right) \\
& +9(q)\left(\frac{8 p+q-1}{2}-8\right)+9(q)\left(\frac{8 p+q-1}{2}-9\right) \\
& \left.+\cdots+9(q)\left(\frac{8 p+q-1}{2}-2 p\right)\right] \\
& =162 p^{2} q+27 p q^{2}-94 p q-5 q^{2}+10 q \\
& { }^{A} \zeta^{c}(G)=\sum_{v \in V(G)} \frac{M(v)}{\varepsilon(v}=2\left[8(q)\left(\frac{8 p+q-1}{2}-1\right)\right. \\
& +18(2 q)\left(\frac{8 p+q-1}{2}-2\right)+27(2 q)\left(\frac{8 p+q-1}{2}-3\right) \\
& \left.+\cdots+27(q)\left(\frac{8 p+q-1}{2}-2 p\right)\right] \\
& +27(q)\left(\frac{8 p+q-1}{2}-4\right)+27(q)\left(\frac{8 p+q-1}{2}-5\right) \\
& +27(2 q)\left(\frac{8 p+q-1}{2}-6\right)+27(2 q)\left(\frac{8 p+q-1}{2}-7\right) \\
& +27(q)\left(\frac{8 p+q-1}{2}-8\right)+27(q)\left(\frac{8 p+q-1}{2}-9\right) \\
& +486 p^{2} q+81 p q^{2}-450 p q-36 q^{2}+117 q .+ \\
& +
\end{aligned}
$$

Theorem 6. Let VPHX[p;q] be the graph of VPhenylenic nanotubes, when $p+1 \leq q \leq 2 p-1$, and $\mathrm{q}$ is even, then we have:

$$
\begin{aligned}
& { }^{E} \zeta^{c}(V P H X[p, q])=54 q^{3}+189 p^{2} q-27 p q^{2}-67 p q+4 q^{2}+14 q \\
& { }^{A} \zeta^{c}(V P H X[p, q])=81 q^{3}+567 p^{2} q-81 p q^{2}-369 p q-171 q^{2}+108 q
\end{aligned}
$$

Proof. In this case the graph has 4 rows and the eccentricity of a vertex in $i^{\text {th }}$ row is same as the eccentricity of a vertex in $(4 \mathrm{p}+1-\mathrm{i})^{\text {th }}$ row where $i=1,2, \ldots, 2 p$. Let $\mathrm{x}_{\mathrm{i}}$ be a vertex in $\mathrm{i}^{\text {th }}$ row. The eccentricity of $\mathrm{x}_{\mathrm{i}}$ is $\varepsilon\left(x_{i}\right)=\left(\frac{8 p+q}{2}-i\right)$ where $i=1,2, \ldots,(4 p-2 p)$. Let $\mathrm{x}_{\mathrm{i}}$ be a vertex in $(4 p-2 q+2 i-1)^{\text {th }}$ or $(4 p-2 q+2 i)^{\text {th }}$ row. The eccentricity of $\mathrm{x}_{\mathrm{i}}$ is $\varepsilon\left(x_{i}\right)=\left(\frac{8 p+q}{2}-(4 p-2 q+i)\right)$ where $i=1,2, \ldots,(q-p)$.

Here the sum of degrees of neighboring vertices in $1^{\text {st }}$ and $4^{\text {th }}$ rows is 6 , whereas the sum of degrees of neighboring vertices in $2^{\text {nd }}$ and $3^{\text {rd }}$ rows is 8 . The product of degrees of neighboring vertices in $1^{\text {st }}$ and $4^{\text {th }}$ rows is 9 , 
whereas the product of degrees of neighboring vertices in $2^{\text {nd }}$ and $3^{\text {rd }}$ rows is 18 . Hence:

$$
\begin{aligned}
& { }^{E} \zeta^{c}(G)=\sum_{v \in V(G)} \frac{S_{v}}{\varepsilon(v)}=2\left[6(q)\left(\frac{8 p+q}{2}-1\right)\right. \\
& +8(2 q)\left(\frac{8 p+q}{2}-2\right)+9(2 q)\left(\frac{8 p+q}{2}-3\right) \\
& +9(q)\left(\frac{8 p+q}{2}-4\right)+9(q)\left(\frac{8 p+q}{2}-5\right) \\
& +9(2 q)\left(\frac{8 p+q}{2}-6\right)+9(2 q)\left(\frac{8 p+q}{2}-7\right) \\
& \left.+9(q)\left(\frac{8 p+q}{2}-8\right)+\cdots+9(q)\left(\frac{8 p+q}{2}-(4 p-2 q)\right)\right] . \\
& +2\left[9(3 q)\left(\frac{8 p+q}{2}-(4 p-2 q+1)\right)\right. \\
& +9(3 q)\left(\frac{8 p+q}{2}-(4 p-2 q+2)\right)+\cdots \\
& \left.+9(3 q)\left(\frac{8 p+q}{2}-(3 p-q)\right)\right] \\
& =54 q^{3}+189 p^{2} q-27 p q^{2}-67 p q+4 q^{2}+14 q \\
& { }^{A} \zeta^{c}(G)=\sum_{v \in V(G)} \frac{S_{v}}{\varepsilon(v)}=2\left[9(q)\left(\frac{8 p+q}{2}-1\right)\right. \\
& +18(2 q)\left(\frac{8 p+q}{2}-2\right)+27(2 q)\left(\frac{8 p+q}{2}-3\right) \\
& +27(q)\left(\frac{8 p+q}{2}-4\right)+27(q)\left(\frac{8 p+q}{2}-5\right) \\
& +27(2 q)\left(\frac{8 p+q}{2}-6\right)+27(2 q)\left(\frac{8 p+q}{2}-7\right) \\
& \left.+27(q)\left(\frac{8 p+q}{2}-8\right)+\cdots+27(q)\left(\frac{8 p+q}{2}-(4 p-2 q)\right)\right] \\
& +2\left[27(3 q)\left(\frac{8 p+q}{2}-(4 p-2 q+1)\right)\right. \\
& \left.+27(3 q)\left(\frac{8 p+q}{2}-(4 p-2 q+2)\right)+\cdots+27(3 q)\left(\frac{8 p+q}{2}-(3 p-q)\right)\right] \\
& =81 q^{3}+567 p^{2} q-81 p q^{2}-369 p q-171 q^{2}+108 q .+
\end{aligned}
$$

Theorem 7. Let VPHX[p;q] be the graph of VPhenylenic nanotubes, when $p+1 \leq q \leq 2 p-1$, and $\mathrm{q}$ is odd, then we have

$$
\begin{aligned}
& { }^{E} \zeta^{c}(V P H X[p, q])=27 q^{3}+189 p^{2} q-27 p q^{2}-94 p q-50 q^{2}+28 q \\
& { }^{A} \zeta^{c}(V P H X[p, q])=27 q^{3}+567 p^{2} q-81 p q^{2}-486 p q-153 q^{2}+171 q
\end{aligned}
$$

Proof. In this case the graph has 4 rows and the eccentricity of a vertex in $i^{\text {th }}$ row is same as the eccentricity of a vertex in $(4 \mathrm{p}+1-\mathrm{i})^{\text {th }}$ row where $i=1,2, \ldots, 2 p$. Let xi be a vertex in $i^{\text {th }}$ row. The eccentricity of $x i$ is $\varepsilon\left(x_{i}\right)=\left(\frac{8 p+q-1}{2}-i\right)$ where $i=1,2, \ldots,(4 p-2 p)$. Let $\mathrm{x}_{\mathrm{i}}$ be a vertex in $(4 p-2 q+2 i-1)^{\text {th }}$ or $(4 p-2 q+2 i)^{\text {th }}$ row. The eccentricity of xi is $\varepsilon\left(x_{i}\right)=\left(\frac{8 p+q-1}{2}-(4 p-2 q+i)\right)$ where $i=1,2, \ldots,(q-p)$.

Here the sum of degrees of neighboring vertices in $1^{\text {st }}$ and $4^{\text {th }}$ rows is 6 , whereas the sum of degrees of neighboring vertices in $2^{\text {nd }}$ and $3^{\text {rd }}$ rows is 8 . The product of degrees of neighboring vertices in $1^{\text {st }}$ and $4^{\text {th }}$ rows is 9 , whereas the product of degrees of neighboring vertices in $2^{\text {nd }}$ and $3^{\text {rd }}$ rows is 18 . Hence

$$
\begin{aligned}
& { }^{E} \zeta^{c}(G)=\sum_{v \in V(G)} \frac{S_{v}}{\varepsilon(v)}=2\left[6(q)\left(\frac{8 p+q-1}{2}-1\right)\right. \\
& +8(2 q)\left(\frac{8 p+q-1}{2}-2\right)+9(2 q)\left(\frac{8 p+q-1}{2}-3\right) \\
& +9(q)\left(\frac{8 p+q-1}{2}-4\right)+9(q)\left(\frac{8 p+q-1}{2}-5\right) \\
& +9(2 q)\left(\frac{8 p+q-1}{2}-6\right)+9(2 q)\left(\frac{8 p+q-1}{2}-7\right) \text {. } \\
& \left.+9(q)\left(\frac{8 p+q-1}{2}-8\right)+\cdots+9(q)\left(\frac{8 p+q-1}{2}-(4 p-2 q)\right)\right] \\
& +2\left[9(3 q)\left(\frac{8 p+q-1}{2}-(4 p-2 q+1)\right)\right. \\
& \left.+9(3 q)\left(\frac{8 p+q-1}{2}-(4 p-2 q+2)\right)+\cdots+9(3 q)\left(\frac{8 p+q-1}{2}-(3 p-q)\right)\right] \\
& =27 q^{3}+189 p^{2} q-27 p q^{2}-94 p q-50 q^{2}+28 q \text {. } \\
& { }^{A} \zeta^{c}(G)=\sum_{v \in V(G)} \frac{M_{v}}{\varepsilon(v)}=2\left[8(q)\left(\frac{8 p+q-1}{2}-1\right)\right. \\
& +18(2 q)\left(\frac{8 p+q-1}{2}-2\right)+27(2 q)\left(\frac{8 p+q-1}{2}-3\right) \\
& +27(q)\left(\frac{8 p+q-1}{2}-4\right)+27(q)\left(\frac{8 p+q-1}{2}-5\right) \\
& +27(2 q)\left(\frac{8 p+q-1}{2}-6\right)+27(2 q)\left(\frac{8 p+q-1}{2}-7\right) \text {. } \\
& \left.+27(q)\left(\frac{8 p+q-1}{2}-8\right)+\cdots+27(q)\left(\frac{8 p+q-1}{2}-(4 p-2 q)\right)\right] \\
& +2\left[27(3 q)\left(\frac{8 p+q-1}{2}-(4 p-2 q+1)\right)\right. \\
& +27(3 q)\left(\frac{8 p+q-1}{2}-(4 p-2 q+2)\right)+\cdots \\
& \left.+27(3 q)\left(\frac{8 p+q-1}{2}-(3 p-q)\right)\right] \\
& =27 q^{3}+567 p^{2} q-81 p q^{2}-486 p q-153 q^{2}+171 q .+
\end{aligned}
$$

Theorem 8. Let VPHX[p;q] be the graph of VPhenylenic nanotubes, when $2 p \leq q$ and $q$ is even, then we have

$$
\begin{aligned}
& { }^{E} \zeta^{c}(V P H X[p, q])=81 p^{2} q+81 p q^{2}-47 p q-15 q^{2}+10 q \\
& { }^{A} \zeta^{c}(V P H X[p, q])=243 p^{2} q+243 p q^{2}-225 p q-162 q^{2}+108 q
\end{aligned}
$$


Proof. In this case the graph has 4 rows and the eccentricity of a vertex in $i^{\text {th }}$ row is same as the eccentricity of a vertex in $(4 \mathrm{p}+1-\mathrm{i})^{\text {th }}$ row where $i=1,2, \ldots, 2 p$. Let $\mathrm{x}$ be a vertex in (2i$1)^{\text {th }}$ or $(2 \mathrm{i})^{\text {th }}$ row. The eccentricity of $x_{i}$ is $\varepsilon\left(x_{i}\right)=\left(\frac{4 p+3 q}{2}-i\right)$ where $i=1,2, \ldots, p .1^{\text {st }}$ and $4^{\text {th }}$ rows is 6 , whereas the sum of degrees of neighboring vertices in $2^{\text {nd }}$ and $3^{\text {rd }}$ rows is 8 . The product of degrees of neighboring vertices in $1^{\text {st }}$ and $4^{\text {th }}$ rows is 9 , whereas the product of degrees of neighboring vertices in $2^{\text {nd }}$ and $3^{\text {rd }}$ rows is 18 . Hence

$$
\begin{aligned}
& { }^{E} \zeta^{c}(G)=\sum_{v \in V(G)} \frac{S_{v}}{\varepsilon(v)}=2\left[6(q)\left(\frac{4 p+3 q}{2}-1\right)\right. \\
& +8(2 q)\left(\frac{4 p+3 q}{2}-1\right)+9(3 q)\left(\frac{4 p+3 q}{2}-2\right) \\
& \left.+9(3 q)\left(\frac{4 p+3 q}{2}-3\right)+\cdots+9(3 q)\left(\frac{4 p+3 q}{2}-p\right)\right] \\
& =81 p^{2} q+81 p q^{2}-47 p q-15 q^{2}+10 q . \\
& { }^{A} \zeta^{c}(G)=\sum_{v \in V(G)} \frac{M_{v}}{\varepsilon(v)}=2\left[8(q)\left(\frac{4 p+3 q}{2}-1\right)\right. \\
& +18(2 q)\left(\frac{4 p+3 q}{2}-1\right)+27(3 q)\left(\frac{4 p+3 q}{2}-2\right) \\
& \left.+27(3 q)\left(\frac{4 p+3 q}{2}-3\right)+\cdots+27(3 q)\left(\frac{4 p+3 q}{2}-p\right)\right] \\
& =243 p^{2} q+243 p q^{2}-225 p q-162 q^{2}+108 q .
\end{aligned}
$$

Theorem 9. Let VPHX[p;q] be the graph of VPhenylenic nanotubes, when $2 p \leq q$, and $q$ is odd, then we have

${ }^{E} \zeta^{c}(V P H X[p, q])=81 p^{2} q+81 p q^{2}-74 p q-15 q^{2}+15 q$

${ }^{A} \zeta^{c}(V P H X[p, q])=243 p^{2} q+243 p q^{2}-306 p q-108 q^{2}+108 q$

Proof. In this case the graph has 4 rows and the eccentricity of a vertex in $i^{\text {th }}$ row is same as the eccentricity of a vertex in $(4 p+1-i)^{\text {th }}$ row where $i=1,2, \ldots, 2 p$. Let xi be a vertex in (2i$1)^{\text {th }}$ or $(2 \mathrm{i})^{\text {th }}$ row. The eccentricity of $x i$ is $\varepsilon\left(x_{i}\right)=\left(\frac{4 p+3 q-1}{2}-i\right)$, where $i=1,2, \ldots, p$. Here the sum of degrees of neighboring vertices in $1^{\text {st }}$ and $4^{\text {th }}$ rows is 6 , whereas the sum of degrees of neighboring vertices in $2^{\text {nd }}$ and $3^{\text {rd }}$ rows is 8 . The product of degrees of neighboring vertices in $1^{\text {st }}$ and $4^{\text {th }}$ rows is 9 , whereas the product of degrees of neighboring vertices in $2^{\text {nd }}$ and $3^{\text {rd }}$ rows is 18 . Hence

$$
\begin{aligned}
& { }^{E} \zeta^{c}(G)=\sum_{v \in V(G)} \frac{S_{v}}{\varepsilon(v)}=2\left[6(q)\left(\frac{4 p+3 q-1}{2}-1\right)\right. \\
& +8(2 q)\left(\frac{4 p+3 q-1}{2}-1\right)+9(3 q)\left(\frac{4 p+3 q-1}{2}-2\right) \\
& \left.+9(3 q)\left(\frac{4 p+3 q-1}{2}-3\right)+\cdots+9(3 q)\left(\frac{4 p+3 q-1}{2}-p\right)\right] \\
& =81 p^{2} q+81 p q^{2}-74 p q-15 q^{2}+15 q . \\
& { }^{A} \zeta^{c}(G)=\sum_{v \in V(G)} \frac{M_{v}}{\varepsilon(v)}=2\left[8(q)\left(\frac{4 p+3 q-1}{2}-1\right)\right. \\
& +18(2 q)\left(\frac{4 p+3 q-1}{2}-1\right)+27(3 q)\left(\frac{4 p+3 q-1}{2}-2\right) \\
& \left.+27(3 q)\left(\frac{4 p+3 q-1}{2}-3\right)+\cdots+27(3 q)\left(\frac{4 p+3 q-1}{2}-p\right)\right] \\
& =243 p^{2} q+243 p q^{2}-306 p q-108 q^{2}+108 q .+
\end{aligned}
$$

\section{Conclusion}

In this work, we extended the eccentric connectivity results for V-Phenylenic nanotube (VPHX[p;q]). We obtained exact values of Ediz eccentric connectivity index and augmented eccentric connectivity index for VPhenylenic nanotube by taking different variation on rows $\mathrm{q}$ and columns $\mathrm{p}$ by calculating sum and product of degrees of neighboring vertices using partitions technique. The generalization of eccentric connectivity index, and exact formulas for these eccentric connectivity indices were obtained.

\section{Acknowledgements}

The authors would like to thank the reviewers for their valuable comments which helped to improve the manuscript.

\section{Orcid:}

Zaheer Ahmad: https://orcid.org/0000-00029860-9038

Muhammad Kamran Jamil:

https://orcid.org/0000-0001-5715-9299

Muhammad Kamran Siddiqui:

https://orcid.org/0000-0002-2607-4847

Muhammad Faisal Nadeem:

https://orcid.org/0000-0002-3175-7191 


\section{References}

[1] M. Randić, J. Amer. Chem. Soc., 1975, 97, 6609-6615.

[2] D.A. Klarner. Polyominoes, In: J.E. Goodman, J.O. Rourke, (eds.) Handbook of Discrete and Computational Geometry. CRC Press, Boca Raton. Chapter 12 (1997) 225242.

[3] N. Trinajstić, Chemical Graph Theory. CRC Press, Boca Raton, FL. (1992).

[4] V. Sharma, R. Goswami, A.K. Mada, J. Chem. Inf. Comput. Sci., 1997, 37, 273-282

[5] S. Gupta, M. Singh, A.K. Madan, J. Math, Anal. Appl., 2002, 266, 259-268.

[6] S. Ediz, J. Optoelectron. Adv. Mater. Rapid Commun., 2010, 4, 1847-1848.

[7] S. Ediz, J. Optoelectron. Adv. Mater. Rapid Commun., 2011, 5, 1263-1264.

[8] H. Dureja, A.K. Madan, Med. Chem. Res., 2007, 16, 331-341.

[9] T. Do slic, M. Saheli, Journal of Mathematical Nano Science., 2011, 1, 25-31.

[10] M.R. Farahani, Int J. Chem Model, 2014, 6, 17-23.

[11] De. Nilanjan, International Journal of Applied Mathematical Research, 2012, 1, 671680.

[12] J. Sedlar, Math. Comput. Chem., 2012, 68, 325-342

[13] Z. Yarahmadi, Iranian Journal of Mathematical Chemistry., 2010, 1, 105-110.

[14] M.R. Farahani, Annals of West University of Timisoara-Mathematics and Computer Science., 2013, 51, 29-37.

[15] M.R. Farahani, World Appl. Sci. J., 2013, 21, 1260-1265.

[16] M.R. Farahani, Journal of Chemica Acta., 2, 2013, 26-31.

[17] M.R. Farahani, Journal of Applied Physical Science International, 2015, 4, 185-190.

[18] M.R. Farahani, W. Gao, International Journal of Applied Mathematics and Machine Learning, 2016, 4, 31-42.

[19] M.R. Farahani, J. Asadpour, M.R.R. Kanna, Asian Academic Research Journal of Multidisciplinary, 2016, 3, 23-29.
[20] M.R. Farahani, M.K. Jamil, M.R.R. Kanna, R. Pradeep Kumar, International Journal of Scientific \& Engineering Research, 2016, 7, 1132-1135.

[21] M.K. Jamil, M.R. Farahani, M.R.R. Kanna, S.M. Hosamani, Journal of Chemical and Pharmaceutical Research, 2016, 8, 80-83.

[22] M.R. Farahani, M.R.R. Kanna, R. Pradeep Kumar, M.K. Jamil, International Journal of Pharmaceutical sciences and Research., 2017, 8, 201-206.

[23] M. Rezaei, A.Q. Baig, W. Sajjad, M.R. Farahani, International Journal of Pure and Applied Mathematics, 2016, 111, 467-477.

[24] W. Gao, M.R. Farahani, M.K. Jamil, Acta Chim. Slov., 2016, 63, 376-379.

[25] W. Gao, W. Wang, M.K. Jamil, M.R. Farahani, Journal of Chemistry, 2016, 1-7.

[26] W. Gao, W.F. Wang, M.K. Jamil, R. Farooq, M.R. Farahani, Bulg. Chem. Commun., 2016, 48, 543-549.

[27] M.K. Jamil, M.R. Farahani, M.R.R. Kanna, The Pharmaceutical and Chemical Journal, 2016, 3, 94-99.

[28] M.R. Farahani, Acta Chimica Slovenica, 2013, 60, 429-432.

[29] Y. Huo, J.B. Liu, A.Q. Baig, W. Sajjad, M. Rezaei, Z. Foruzanfar, M.R. Farahani, J. Comput. Theor Nanos., 2017, 14, 1832-1836.

[30] N.P. Rao, K.L. Lakshmi, Dig. J. Nanomater. Bios., 2010, 6, 81-87.

[31] V. Alamian, A. Bahrami, B. Edalatzadeh, Int. J. Mol. Sci., 2008, 9, 229-234.

[32] M. Alaeiyan, A. Bahrami, M.R. Farahani, Digest. J. Nanomater. Bios., 2011, 6, 143-147.

[33] J. Asadpour, Adv. Mater.-Rapid Commun., 2011, 5, 769-772.

[34] A. Bahrami, J. Yazdani, Digest. J. Nanomater. Bios., 2009, 4, 141-144.

[35] M. Davoudi Mohfared, A. Bahrami, J. Yazdani, Digest. J. Nanomater. Bios., 2010, 5, 441-445.

[36] M.R. Farahani, Int. J. Chem Model., 2013, 5, 479-484.

[37] M.R. Farahani, Acta Chimica Slovenica., 2013, 60, 429-432. 
[38] M.R. Farahani, Int. J. Theoretical Chemistry, 2013, 1, 01-09.

[39] M.R. Farahani, M.R. Rajesh Kanna, Journal of Chemical and Pharmaceutical Research, 2015, 7, 241-245.

[40] V. Kumar, A.K. Madan, J. Math. Chem., 2006, 39, 511-521.

[41] V. Kumar, A.K. Madan, J. Math. Chem., 2007, 42, 925-940.

[42] V. Lather, A.K. Madan, Croat. Chem. Acta., 2005, 78, 55-61.

[43] S. Sardana, A.K. Madan, J. Mol. Struct., (Theochem), 2003, 638, 41-49.

[44] S. Sardana, A.K. Madan, J. Mol. Model., 2002, 8, 258-265.

[45] H. Dureja, S. Gupta, A.K. Madan, J. Mol. Graph. Model, 2008, 26, 1020-1029.

[46] S. Gupta, M. Singh, A.K. Madan, J. Math. Anal. Appl., 2002, 275, 386-401.
[47] V. Kumar, S. Sardana, A.K. Madan, J. Mol. Model, 2004, 10, 399-407.

[48] S. Sardana, A.K. Madan, Math. Comput. Chem., 2001, 43, 85-98.

[49] H. Dureja, A.K. Madan, Int. J. Pharm., 2006, 323, 27-33.

[50] S. Gupta, M. Singh, A.K. Madan, J. Math. Anal. Appl., 2002, 266, 259-268.

[51] A. Ilic, I. Gutman, Math. Comput. Chem., to appear.

[52] N.P. Rao, K.L. Lakshmi, Digest Journal of Nanomaterials and Biostructures, 2010, 6, 8187.

How to cite this article: Zaheer Ahmad, Maria Naseem, Muhammad Kamran Jamil, Muhammad Kamran Siddiqui, Muhammad Faisal Nadeem. New results on eccentric connectivity indices of $\mathrm{V}$ Phenylenic nanotube. Eurasian Chemical Communications, 2020, 2(6), 663-671. Link: http://www.echemcom.com/article_104851.html 